\title{
Research and Strategy of Urban Traffic Congestion Control
}

\author{
Zhiran Wang*
}

Matt City Construction Engineering Limited. E-mail: zhiran@qq.com

Abstract: With the acceleration of urbanization, urban public transportation has been developed and improved for a long time as well. Currently, China's traditional and single ground transportation system has been transformed into a multi-functional and compound multi-transportation one. However, the congestion problem in cities has become increasingly serious. Cities in different countries should take different measures to implement the accumulation pole. They also should focus on energy source consumption, environmental pollution and health care brought by traffic congestion. The practice and research countermeasures of relieving urban traffic congestion can be divided into developmental, managerial and restrictive measures. Urban traffic congestion is a systematic problem, which needs to be treated by comprehensive measures, and given priority to the use of developmental measures in order to improve urban traffic supply capacity. It is necessary to strive to enhance urban traffic management level, practice administrative measures. With historical basis, development level and fairness of urban development in China need to be taken into account, and carefully consider the use of restrictive measures.

Keywords: Urban Transportation; Traffic Congestion; Government Governance Countermeasures

\section{Introduction}

In the process of building a service-oriented government ruled by law, it is necessary to promote the modernization of the national governance system and governance capacity, which is the key to comprehensively deepening the system reform and transforming the government governance concept. In China, urban traffic problems have a long history, with traffic congestion and traffic accidents emerging one after another. Public traffic problems and parking problems become increasingly prominent, resulting in increasingly acute social problems and contradictions. This kind of situation is not only restrict the economic development and urbanization process, but also challenge the government's governance capacity and service efficiency. The World Bank predicts that the global urbanization rate will reach $55 \%$ by 2020 , which means that more than half of the world's people will live in cities. According to the data released by the National Bureau of Statistics, the urbanization rate in China reached $57.35 \%$ in 2016, and the urban population increased significantly. As an advanced form of productivity organization and lifestyle, cities naturally have the features of attracting a large number of people, industries and resources. Cities are areas with most concentrated population and economic and social activities. The continuous growth of urban residents' demand for mobility leads to the rapid growth of public transportation and private transportation. However, there is a lot of money being spent on cities' congestion control, but the effect is still relatively limited. Traffic congestion has multidimensional causes, and in this case, local and short-term governance cannot really solve the problem

Copyright (C) 2020 Zhiran Wang

doi: $10.18686 /$ utc.v6i2.87

This is an open-access article distributed under the terms of the Creative Commons Attribution Non-Commercial License

(http://creativecommons.org/licenses/by-nc/4.0/), which permits unrestricted non-commercial use, distribution, and reproduction in any medium,

provided the original work is properly cited. 
by itself. Therefore, combing the multi-dimensional strategies of congestion control is of great significance for understanding traffic congestion and seeking suitable control measures.

\section{Characteristics and comprehen- sive impact of traffic congestion}

\subsection{China's urban traffic congestion charac- teristics}

With the process of urbanization and motorization in China, the traffic congestion in various cities has been accelerated. Under the condition of limited road infrastructure and traffic management, the continuous increase in the number of cars has become the main cause of congestion. From 1991 to 2011, the number of private cars increased from 910,400 to $73,267,900$, with an average annual growth rate of $24.56 \%$. At present, China's urban traffic congestion is of diversified characteristics.

(1) Traffic congestion is normalized, gradually spreading from the central city to the periphery of the urban area, and the congestion time and scope are constantly expanding;

(2) In the morning and evening peak hours, the main commuter roads are seriously congested, and most of them are traffic congestion in one direction;

(3) The congestion of some road network nodes and intersections restricts the traffic operation of the road network, and spreads from "point" congestion to "point, line and side" congestion;

(4) The traffic order is chaotic, and the mixed traffic reduces the road capacity.

\subsection{Comprehensive impact of traffic conges- tion}

Traffic congestion leads to time and energy consumption, reduces production efficiency, and brings environmental pollution and health problems. It is estimated that the economic loss caused by traffic congestion accounts for about $2 \% \sim 3 \%$ of GDP. In 2002, traffic congestion in 75 metropolitan areas of the United States caused economic losses of 63.2 billion US dollars, with an average per capita of about 829 US dollars, while the annual losses caused by traffic congestion in Tokyo amounted to 12 trillion yen. According to the data of Texas Transportation Research Institute in 2011, the av- erage delay time of every passer-by is 38 hours, with a total delay time of 5.5 billion hours, consuming 2.9 billion gallons of fuel and causing a loss of 121 billion US dollars. In addition, traffic congestion increases air pollution, and leads to the increase of travel time and distance dependent on motor vehicles, which easily leads to health problems such as respiratory diseases and obesity.

\section{Analysis of factors and causes af- fecting urban traffic congestion}

With the improvement of urbanization level and the expansion of urban scale, urban traffic congestion is becoming normal, diversified and complicated, and its congested areas gradually expand from urban areas to suburbs, from old cities to new development areas. Therefore, under the background of building a service-oriented government and building a harmonious society, urban scale, population, number of cars and road planning level are important indicators that affect urban traffic congestion. Only by fundamentally analyzing the root causes of urban traffic congestion can we realize a smooth traffic, convenient travel and safe and comfortable urban transportation system.

\subsection{Unreasonable city planning}

As the leader of urban construction and management, urban planning is not only related to the future development and overall spatial layout of the city, but also related to the comprehensive deployment of urban planning, urban construction and urban operation. As a part of urban planning, urban transportation planning and municipal construction planning can only be carried out on the basis of the overall spatial layout of urban planning. Therefore, the unreasonable urban planning will inevitably lead to the imperfect urban transportation planning system, resulting in the hidden dangers of traffic congestion in the planning, design, layout and operation of various transportation facilities.

\subsection{Lagging construction of new transporta- tion infrastructure}

Generally speaking, unreasonable land use layout, inefficient traffic management, expansion of car ownership, and time-space agglomeration of car use have caused traffic supply to lag behind traffic demand, which has become an important reason for congestion. In Chi- 
nese cities where urban space is changing rapidly, the factors and causes of traffic congestion are different.

\subsection{Imperfect urban transportation planning system}

Sun Zhe believes that urban transportation planning is the foundation of establishing urban transportation system, and the traffic congestion problem in most cities is caused by the one-sided and unreasonable transportation planning and design, and the failure to look at the overall and comprehensive development of a city from a long-term perspective. It not only wastes the limited public resources, but also makes the transportation resources unable to exert the maximum social utility, which is not conducive to the fair and effective allocation of transportation resources, resulting in traffic congestion and traffic accidents. Experts believe that due to historical and political reasons, most urban planning is a single regional city at the beginning. However, with the expansion of urban population, urban roads must be expanded and extended outward. However, the traditional urban traffic layout has become a system, and unified design and planning cannot be carried out in a short time, resulting in serious traffic congestion.

\subsection{Less mode of travel}

Green travel modes such as walking and cycling are shrinking day by day, which increases the pressure of urban transportation system. However, traffic congestion in different cities presents different characteristics and dynamic mechanisms. Therefore, recognizing the causes of congestion, and then "prescribing the right medicine" is an effective strategy to control congestion.

\section{Study of urban traffic congestion control countermeasures}

With the acceleration of urbanization and the improvement of industrialization level, the population size of the city will continue to expand. In the case of limited urban capacity and insufficient public resources, only unified planning and effective integration of various transportation resources, and administrative restrictive measures should be taken to speed up the construction of public transportation, improve the modern governance level of public transportation, and coordinate the intelligent management of public transportation with manual management, so as to realize the optimization of the utilization efficiency of public transportation resources.

\subsection{Rational planning of urban traffic and improvement of utilization rate of the public transport}

Yong Guozhong believes that with the acceleration of the pace of "cars entering the family", the early urban planning, urban functional location division and urban transportation system can no longer meet the needs of industrialization and modernization. The traditional single-center pattern construction, in which the living area, administrative area, industrial area and leisure area are planned separately, will easily lead to the urban network pattern of "spreading big cakes". In the process of constructing transportation system, we should combine transportation function, commercial function and service function, change the single-center service pattern of urban transportation, actively create multi-functional and comprehensive multi-center service areas, and speed up the construction of supporting public facilities. Zhou Jun and $\mathrm{Su}$ Yunting believe that in the process of planning traffic layout, all high-quality public resources should be avoided to be concentrated in a single area, and the urban spatial layout and public resources distribution structure should be developed in the direction of equalization, diversification and convenience, satellite cities should be built, the traffic strategic layout of urban sub-centers should be improved, planning disconnection and single-center traffic network pattern should be avoided, and traffic congestion in main urban areas and main roads should be alleviated.

\subsection{Traffic congestion management based on spatial structure}

Urban spatial structure is closely related to fic behavior. Specific urban form requires specific traffic modes to adapt, while traffic is reshaping spatial form. The traffic performance of single-center and multi-center spatial structures has become a hot spot. On the one hand, the single-center structure increases the traffic pressure in the central area of the city, but it is beneficial to the development of public transportation. The multi-center spatial structure can improve the spatial matching degree of residence, employment and public facilities, thus improving commuting structure, reducing traffic demand and relieving traffic congestion. Especially when the 
average travel distance and time are beyond the acceptable range, the effect of local governance is very limited. Only by changing from single-center structure to multi-center layout can the traffic congestion be effectively solved.

\subsection{To standardize and improve traffic laws and regulations to promote citizens' civilized travel}

Traffic congestion is not only the normal state of urbanization, but also a difficult problem of government governance. Therefore, under the background of building a service-oriented government and a government ruled by law, it is necessary to improve not only the government's service level and management efficiency, but also the citizens' comprehensive quality and public moral cultivation, so as to realize the smooth progress of government governance of public traffic congestion. Yang Xiangqian believes that in most public traffic accidents, citizens' driving behavior is irregular, especially drunk driving, fatigue driving and overload driving are the main causes of public traffic accidents. On the one hand, standardizing the relevant laws on public transportation, advocating civilized driving and safe travel are the key to reduce traffic congestion and traffic accidents. On the other hand, the government should increase penalties for illegal drivers, deal with all kinds of illegal driving behaviors strictly and severely, and ensure the order of public transportation and the fairness and authority of laws.

\subsection{Traffic congestion control based on land use}

There is a close relationship between land use and transportation system. The type, intensity and spatial connection of land use greatly affect the frequency, time and choice of transportation mode of residents, while the transportation system affects the spatial accessibility of land use, which gradually changes the value, type and scale of land. It is difficult to effectively solve the congestion problem by simply increasing the supply of traffic capacity, and it is necessary to coordinate the management of traffic demand, traffic layout and land use. Among them, the bus-oriented development mode integrating transportation and land use has become an important direction. Areas with high population density are usually conducive to the use of non-motorized transportation and public transportation. If mixed and compact land development is adopted, the internalization of transportation demand can be effectively realized, the use ratio of non-motorized transportation and public transportation can be increased, and motor vehicles can be reduced.

\section{Conclusion}

From the perspective of economics, traffic congestion is a kind of traffic loss when traffic supply cannot meet traffic demand. With the rapid growth of urban population, the continuous expansion of urban space and the rapid growth of the number of motor vehicles, the problem of traffic congestion in urban areas of big cities has become increasingly prominent. The bottleneck of road infrastructure, the relatively lagging development of public transportation and the concentration of peak trips of residents have become the main factors restricting the smooth flow. Cities have great attraction to population, industry and resources, and a large number of population and economic production activities gather in a limited space. Under the specific traffic demand situation (morning and evening tidal traffic demand), the time imbalance and space imbalance between traffic supply and demand are particularly prominent, and urban congestion in peak period becomes a normal state. Traffic congestion has become a persistent problem in the governance of contemporary big cities. The causes of traffic congestion are complex, which involve many factors such as urban history, urban function orientation and urban industrial layout. Therefore, the problem of urban traffic congestion is a systematic problem, which needs to be treated by comprehensive measures.

\section{References}

1. Lei Y, Huang C. Literature review and management expectation on the alleviating urban traffic congestion. China Transportation Review 2018; 40(4): 8$11,42$.

2. Wu X, Wu D, Guan S, et al. Survey of urban traffic congestion. Popular Science \& Technology 2015; 17(8): 151-155.

3. Lin X, Yang J. Multidimensional reviews on characteristics and solutions of urban congestion. China Transportation Review 2015; 37(8): 55-61. 